\title{
Application of Weibull Distribution for Detection and Identification of Partial Discharge in XLPE Cable
}

\author{
Xiaoli Hu, a , Jun Yong ${ }^{2}$, Bo Yang ${ }^{2}$, Minglin $\mathrm{Liu}^{2}$, Hailei Meng ${ }^{2}$, Xiaobin Sun ${ }^{2}$, \\ Yubing Duan ${ }^{1}$, Xinyan Feng ${ }^{3}$, Yongpeng $\mathrm{Xu}^{4, \mathrm{~b}}$ \\ ${ }^{1}$ Shandong Elecrtic Power Research Institute, Jinan, 250002, China \\ ${ }^{2}$ State Grid Shandong Electric Power Company, Ji'nan, 250002, China \\ ${ }^{3}$ State Grid Shandong Electric Power Maintenance Company, Ji'nan, 250000, China \\ ${ }^{4}$ Departments of Electrical Engineering, Shanghai Jiao Tong University, 200240, China \\ ahuxl_cn@sina.com, bypxu1990@qq.com
}

Keywords: Weibull distribution, partial discharge, XLPE cable

Abstract. In order to study the application of Weibull distribution in cable partial discharge pattern recognition. We apply a $10 \mathrm{kV} 30 \mathrm{~m}$ XLPE cable for this study. Then design four kinds of typical artificial defect model and analyze each of defect model's discharge pulse signals. Finally, estimate the Weibull distribution of signals by use of the maximum likelihood method. The results show that Weibull probability distribution is similar to partial discharge amplitude distribution, the shape parameter $\beta$ can be used to identify different types of discharge.

\section{Introduction}

Partial discharge is an important cause of cable aging insulation. It's closely related to the breakdown process of the cable insulation material. Therefore, research on partial discharge characteristics is meaningful for improving the operational life of cable.

The research shows that partial discharge process is a random process. We can study the process by use of statistics. The cumulative probability distribution of a single type of partial discharge's amplitude distribution is in line with the two-parameter Weibull distribution. Two types of mixing discharge is in line with five-parameter mixing Weibull distribution[1,2]. On this basis, the author conducted experiments on several typical artificial defects of cable and obtained different pulse amplitude distribution. Then study the application of two-parameter Weibull distribution in discharge pattern recognition.

Weibull distribution method. Weibull distribution is the theoretical basis of reliability analysis and life testing. Weibull distribution includes single parameter, two parameter, three parameter, mixing Weibull distribution and other forms.

Two-parameter Weibull distribution is determined by shape parameter $\alpha$ and scale parameter $\beta$. Among them, shape parameter $\alpha$ determines the basis shape of distribution density curve. scale parameter $\beta$ plays the role of enlarge or reduce the curve and it doesn't influence distribution shape. Changing the shape parameter can indicate the failure conditions of different stages. It can also be used as an approximate normal, lognormal, exponential distribution, etc.

For pulse signal generated by the single PD source. We can use $\mathrm{W}(\alpha, \beta)$ to indicate two-parameter Weibull distribution. Its distribution function was shown as equation (1). $\alpha>0, \beta>0, \mathrm{q}_{\mathrm{m}}$ represents the detected discharge signal, represents measuring instrument sensitivity[3,4] .

$$
\begin{aligned}
& F\left(q_{i}\right)=1-\exp \left[\left(-\left(\frac{q_{i}}{\alpha}\right)^{\beta}\right]\right. \\
& f\left(q_{i}\right)=\frac{\beta}{\alpha}\left(\frac{q_{i}}{\alpha}\right)^{\beta-1} \exp \left[\left(-\left(\frac{q_{i}}{\alpha}\right)^{\beta}\right]\right.
\end{aligned}
$$

Clem test (equation 2) can be used to test the fit. Among them, $\mathrm{N}$ represents pulse number, $\mathrm{X}$ represents discharge pulse amplitude. Generally, $\mathrm{W}^{2}<0.5$ examined while $\mathrm{W}^{2}>0.5$ refused fitting 
results. Using fitting out of the $\alpha$ and $\beta$ can calculate the number of discharges per cycle, the average discharge amplitude per cycle, the average amplitude per discharge and other parameters

$$
W^{2}=\frac{1}{12 N}+\sum_{i=1}^{N}\left(F\left(X_{(i)}\right)-\frac{2 i-1}{2 N}\right)^{2}
$$

Experiment design. In order to study the relationship between Weibull distribution and pulse amplitude statistical properties of medium (low) voltage XLPE cable partial discharge. Regard a 30m $10 \mathrm{kV}$ XLPE cable as object. Designed needle corona, suspended electrode, acupuncture treatment and cut four defects. (shown figure 1). Each of defects was designed three test model specifications.
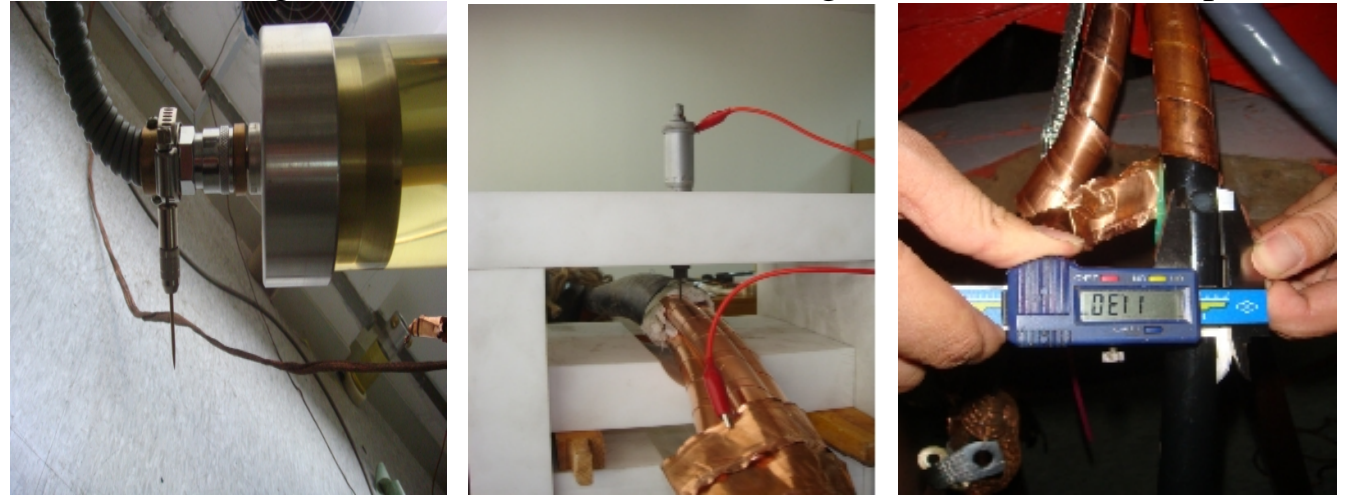

Fig 1.the test site map of Needle, acupuncture treatment, cuts

The method of partial discharge pulse measurement is according to pulse current method of IEC60270. Testing process: firstly, increasing the voltage until cable produces PD. After maintaining the voltage 5 minutes down to zero and then increasing the voltage until producing PD. Maintaining the voltage 1 minutes and then recording data. For each of model, voltage is applied for about 20 minutes. PD meter can record and analyze 4000 frequency cycle discharge pulse amplitude.

(1) Cable core needle corona model

Installed tip model at cable terminal core conductor. Tip radius of curvature of the model is $50 \mu \mathrm{m}$, $100 \mu \mathrm{m}, 150 \mu \mathrm{m}$. The length is $50 \mathrm{~mm}$.It was hung on the cable conductor. In air, single needle corona only produces negative pulse. The discharge amount is small and concentrated. It produces discharge when voltage was increased to $2 \mathrm{kv}, 2.5 \mathrm{kV}, 4 \mathrm{kV}$ respectively. The discharge amount is $15 \mathrm{pC}, 21 \mathrm{pC}$, $32 \mathrm{pC}$ respectively.

(2)Cable suspended electrode model

Installed round copper electrode at cable terminal as suspended electrode. The round copper's diameter is respectively $16 \mathrm{~mm}, 14 \mathrm{~mm}, 12 \mathrm{~mm}$. The thickness is $2 \mathrm{~mm}$. All of them state discharging when the voltage reaches $13 \mathrm{kV}$. The measured discharge amount is about $100 \mathrm{pC}$ after reaching a stable discharge.

(3)The cable body acupuncture treatment model

Steel whose tip radius of curvature needles into the cable body. Pierce the outer insulation, copper, outer semiconductor layer. The depth of tie into XLPE insulation layer is respectively $2 \mathrm{~mm}, 2.5 \mathrm{~mm}$ and $3 \mathrm{~mm}$. applied voltage is respectively $8 \mathrm{kV}, 5 \mathrm{kV}$ and $4 \mathrm{kV}$ when discharge appears. The discharge amount is respectively $20 \mathrm{pC}, 42 \mathrm{pC}$ and $65 \mathrm{pC}$.

(4)Cut the cable body hybrid model

Cut out the irregular shape on cable body as insulation damage. Cutting the outer semiconducting layer with knife and damaging XLPE insulation layer. Scratching shape is depth $1.3 \mathrm{~mm}$, length $12.1 \mathrm{~mm}$, width $11.3 \mathrm{~mm}$, depth $2.1 \mathrm{~mm}$, length $12.8 \mathrm{~mm}$, width $12.4 \mathrm{~mm}$ and depth $3 \mathrm{~mm}$, length $18.8 \mathrm{~mm}$, width $14.8 \mathrm{~mm}$. The initial discharge voltage is respectively $8 \mathrm{kV}, 7 \mathrm{kV}$ and $4 \mathrm{kV}$. The discharge amount is respectively is respectively $200 \mathrm{pC}, 400 \mathrm{pC}$ and $500 \mathrm{pC}$.

Experimental results and analysis. Due to a variety of different types of discharge pulse amplitude, Weibull distribution parameter could be used to indicate different types of discharge. We analyze the measured discharge amplitude of four aforementioned models and fit discharge amplitude by use of Weibull probability function. We also estimated parameter by use of maximum probability method and inspect estimated results by use of Cramer's rule. 
As can be seen from the figure, the partial discharge pulse amplitude distribution of different types of cable is significantly different. Needle model belongs to single point corona model, the initial discharge voltage and discharge amount increase with the addition of tip radius of curvature. Since it's easier to produce corona under negative polarity than positive, there is only negative discharge pulse. Because of this reason, we didn't fit positive pulse. Itsvalue of $\beta$ is more than 30 (figure 2). Discharge signals can be detected in the positive and negative half-cycle. The value of $\alpha$ is significantly different between positive and negative. But the value of $\beta$ is between 4 to 10 (figure 2). Acupuncture treatment trials belong to internal insulation defects. Discharge pulse under positive polarity or negative polarity all can be measured. Its value of $\beta$ is between 10 to 30 (figure 2). Due to cuts is seriously damaged to insulation, the discharge amount is large, normally more than $100 \mathrm{pC}$. After fitting, its value of scale parameter $\beta$ is small, generally around 1 (figure 2).

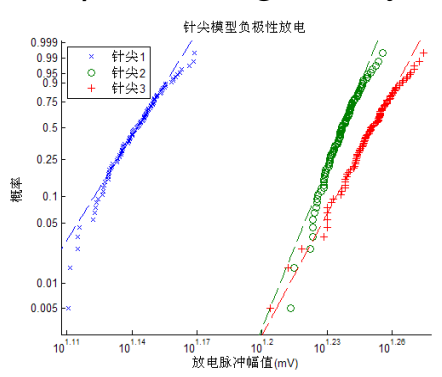

(a)

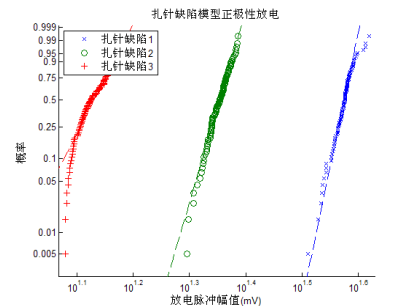

(d)

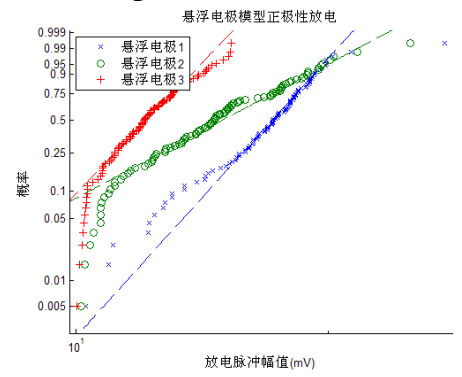

(b)

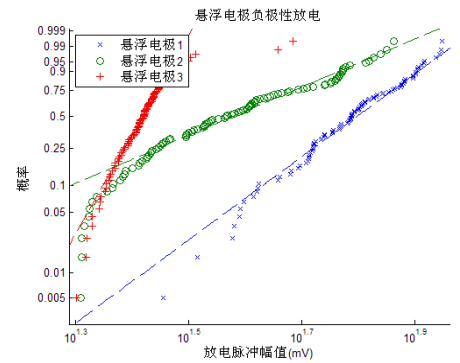

(c)

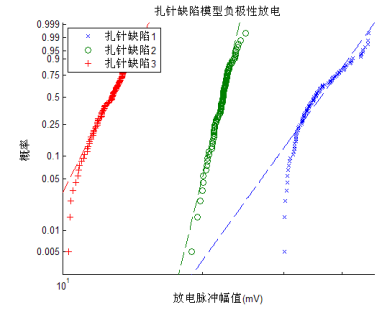

(e)

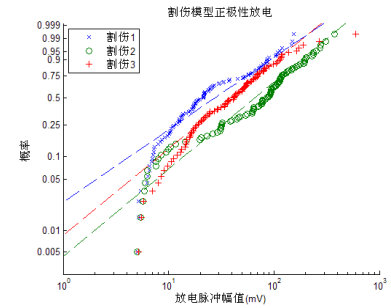

(f)

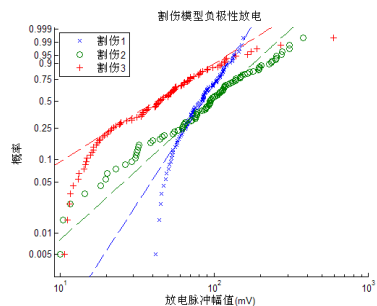

(g)

Fig 2. Weibull distribution of Needle model negative discharge, suspended model positive and negative discharge, acupuncture treatment defects positive and negative discharge, cutting positive and negative discharge

It's can be seen that a single type of discharge's $\mathrm{W}^{2}$ is smaller than 0.5 after inspecting by Cramer's rule. It represents that the pulse amplitude distribution is similar to two-parameter Weibull distribution. The same type of discharge has same value of shape parameter $\beta$ while there are significant differences comparing to other types of discharge. The differences are obvious. Therefore, it can be used as the rules of different discharge pattern recognition. 
Tab.1 Weibull distribution of different models

\begin{tabular}{|c|c|c|c|c|c|}
\hline defects & polarity & classification & $\alpha$ & $\beta$ & $\mathrm{W}^{2}$ \\
\hline \multirow[t]{6}{*}{ needle } & + & 1 & $\mathrm{X}$ & $\mathrm{X}$ & $\mathrm{X}$ \\
\hline & & 2 & $\mathrm{X}$ & $\mathrm{X}$ & $\mathrm{X}$ \\
\hline & & 3 & $\mathrm{X}$ & $\mathrm{X}$ & $\mathrm{X}$ \\
\hline & - & 1 & 14.10 & 43.14 & 0.06 \\
\hline & & 2 & 17.39 & 56.82 & 0.14 \\
\hline & & 3 & 17.85 & 30.99 & 0.25 \\
\hline \multirow[t]{6}{*}{ Suspended electrode } & + & 1 & 17.92 & 6.61 & 0.44 \\
\hline & & 2 & 16.07 & 5.20 & 0.18 \\
\hline & & 3 & 12.77 & 9.43 & 0.21 \\
\hline & - & 1 & 72.05 & 6.54 & 0.22 \\
\hline & & 2 & 34.35 & 4.76 & 0.12 \\
\hline & & 3 & 29.22 & 5.81 & 0.48 \\
\hline \multirow[t]{6}{*}{ Acupuncture treatment } & + & 1 & 38.15 & 25.64 & 0.32 \\
\hline & & 2 & 22.94 & 26.49 & 0.04 \\
\hline & & 3 & 14.07 & 11.82 & 0.46 \\
\hline & - & 1 & 37.94 & 8.49 & 0.49 \\
\hline & & 2 & 22.62 & 22.02 & 0.14 \\
\hline & & 3 & 12.52 & 11.21 & 0.17 \\
\hline \multirow[t]{6}{*}{ cuts } & + & 1 & 37.58 & 1.10 & 0.48 \\
\hline & & 2 & 99.65 & 1.20 & 0.17 \\
\hline & & 3 & 58.84 & 1.08 & 0.15 \\
\hline & - & 1 & 94.83 & 3.39 & 0.15 \\
\hline & & 2 & 119.58 & 1.53 & 0.10 \\
\hline & & 3 & 68.12 & 1.16 & 0.28 \\
\hline
\end{tabular}

Conclusion. The tests show that the value of fitting Weibull distribution parameter of cable partial discharge pulse amplitude distribution is related to the discharge phenomenon. The scale parameter $\beta$ is different for different polarity and types of discharge. It can be used as the rules of pattern recognition for different types of discharge. Different types of discharge have different Weibull distribution of positive and negative pulse amplitude. Compared with the parameter $\beta$ of single polarity pulse, it's more credible. It can be used as the basis for classification of partial discharge.

\section{References}

[1] Tang Ju, et al. Application of the Weibull Distribution for the Recognition of Transformer PD Phenomena. Journal of Chongqing University (Natural Science Edition), 2002(04).

Reference to a book:

[2] Bu Ke-wei, et al. Application of the Weibull Distribution to Recognition of GIS PD Phenomena. High Voltage Apparatus, 2009(03).

[3] Contin, A., et al. Weibull approach to the investigation of partial discharges in aged insulation systems. 1996. Montreal, Can: IEEE. 
[4] Contin, A., M. Cacciari, and G.C. Montanari. Estimation of Weibull distribution parameters for partial discharge inference. 1994. Arlington, TX, USA: IEEE. 